\title{
A SAR Processing Algorithm for TOPS Imaging Mode Based on Extended Chirp Scaling
}

\author{
Pau Prats, Rolf Scheiber, Josef Mittermayer, \\ Adriano Meta, and Alberto Moreira \\ German Aerospace Center (DLR), Microwaves and Radar Institute \\ P.O. Box 1116, D-82234, Wessling, Germany \\ Email: Pau.Prats@dlr.de
}

\author{
Jesus Sanz-Marcos \\ Universitat Politècnica de Catalunya (UPC) \\ Signal Theory and Communications \\ Barcelona E-08034, Spain \\ Email: jesus_sanz@mac.com
}

\begin{abstract}
This paper presents an efficient phase preserving processor for TOPS (Terrain Observation by Progressive Scans) imaging mode. TOPS has been proposed as a new wide swath imaging mode, which solves the problems of scalloping and azimuth-varying ambiguities introduced by the conventional ScanSAR mode by means of steering the antenna along the azimuth direction. An algorithm based on ECS (Extended Chirp Scaling) is proposed, which uses sub-apertures and a new azimuth scaling step. The proposed solution is also efficient in the sense that it allows selecting the final azimuth image spacing by means of azimuth scaling, hence easing the forthcoming mosaicking of the different subswaths. Simulations with point targets are used to validate the processor.
\end{abstract}

\section{INTRODUCTION}

Large swath coverage is an essential requirement by a number of applications. The standard ScanSAR mode achieves this requirement by periodically switching the antenna elevation beam to point at different range subswaths [1], [2], hence acquiring a certain number of bursts per subswath. The tradeoff is the azimuth resolution loss due to the reduction of the observation time of targets. However, the ScanSAR mode has some disadvantages besides resolution loss: scalloping (periodical modulation of the amplitude in the focused image), and azimuth-varying ambiguity ratio and noise equivalent sigma-zero $\left(\sigma_{0}\right)$. They are a consequence of the fact that different targets are observed under different portions of the azimuth antenna pattern. In order to reduce these effects, different bursts are incoherently averaged, with the consequent further worsening of the azimuth resolution.

TOPS (Terrain Observation by Progressive Scans) has been proposed as a new wide swath imaging mode [3]. It overcomes the problems of scalloping and azimuth-varying ambiguities introduced by the conventional ScanSAR mode by means of steering the antenna along-track. The solution proposed in [3] to achieve the same swath coverage and avoid the undesired effects of ScanSAR consists in rotating the antenna throughout the acquisition from backward to forward at a constant rotation speed $\omega_{\text {r }}$ (see Fig. 1), opposite to the spotlight case, resulting in the opposite effect, i.e. a worsening of the azimuth resolution. However, all targets are observed under the same azimuth antenna pattern, and therefore the scalloping effect disappears and azimuth ambiguities and signal-to-noise ratio become constant in azimuth. At the end of the burst, the

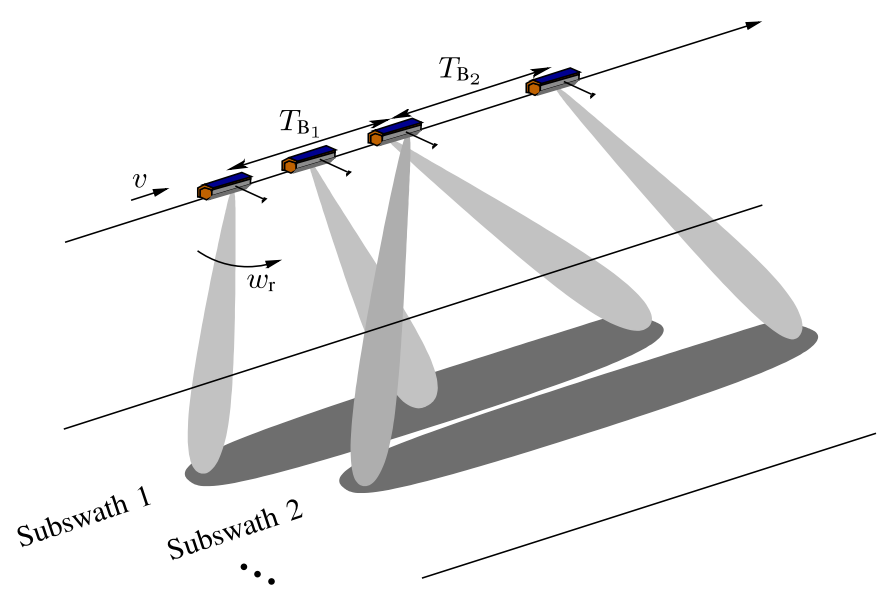

Fig. 1. Acquisition geometry of the TOPS imaging mode.

antenna look angle is changed to illuminate a second subswath, pointing again backwards. When the last subswath is imaged, the antenna points back to the first subswath, so that no gaps are left between bursts of the same subswath.

Standard processing approaches for other imaging modes (Stripmap, Spotlight, ScanSAR) are not valid for the TOPS mode. Only with pre- and post-processing approaches these algorithms can be used with data acquired in the TOPS mode, with the consequent increase in the computation burden. Therefore, a dedicated processor must be developed if efficient focusing is desired. Section II first analyzes the TOPS signal characteristics and comments the limitations of some possible approaches. The proposed processor is expounded in Section II-C. Section III shows some results with point targets, while Section IV presents the conclusions.

\section{TOPS PROCESSING}

\section{A. TOPS signal characteristics}

The TOPS raw data signal in one burst has similarities with both ScanSAR and spotlight ones. The TOPS signal resembles the spotlight signal in the sense that the scene bandwidth is larger than the pulse repetition frequency (PRF). Also, it has similarities with the ScanSAR mode in the sense that the burst duration is shorter than the output focused burst. The 


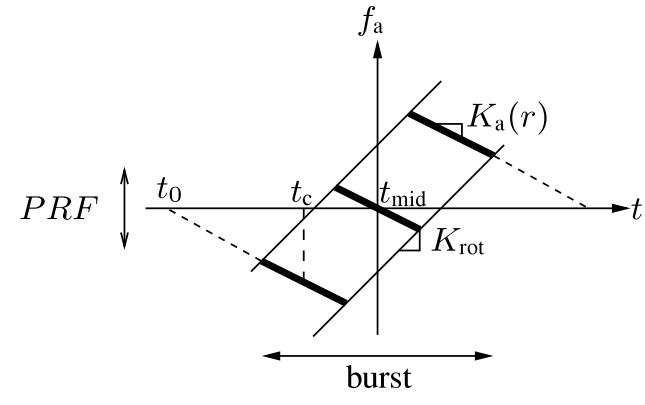

Fig. 2. Time-frequency diagram in the TOPS imaging mode.

signal properties can be clearly visualized by means of a timefrequency diagram. Fig. 2 shows the Doppler history of three targets at the same range position (thick solid lines), but with different azimuth positions. The abscissas correspond to the azimuth time and the ordinates to the instantaneous frequency. The target at the beginning of the burst is observed under a negative squint angle, hence resulting in negative Doppler frequencies. On the other hand, the target at the end of the burst has positive Doppler frequencies. Usually, the total scene bandwidth spans over several times the PRF, similar as it occurs with the spotlight mode. Consequently, some procedure is necessary to account for this insufficient sampling of the azimuth signal. Concerning the similarities with ScanSAR, consider the first target depicted in Fig. 2. Although it is observed in the middle of the beam at the time instant $t_{\mathrm{c}}$ (i.e. beam-center time), after the focusing the target should appear at zero-Doppler position $t_{0}$. Hence, the output focused burst is larger than the burst duration.

\section{B. Limitations of Existing Approaches}

In the original reference where the TOPS imaging mode is proposed also an experimental processor is presented [3]. The proposed processor uses a pre-processing approach to accommodate for the larger scene bandwidth as presented in [4]. Afterward, data are focused using a wavenumber domain processor. In order to carry out the unfolding in time domain, a post-processing approach based on a multirate filter bank is applied, as described in [1]. The only drawback of this approach is the need of pre- and post-processing steps, which increases the computation burden.

The processing approach used for ScanSAR presented in [2] can be also a possibility, together with the sub-aperture approach to have an instantaneous bandwidth smaller than the PRF, as proposed in [5]. For each sub-aperture the signal is properly sampled, so that range cell migration correction (RCMC) is carried out using ECS. However, the azimuth processing of [2] uses azimuth scaling + SPECAN instead of conventional matched filtering to take into account the scene duration, which is larger than the burst duration. This approach consists in first substituting the hyperbolic azimuth phase by a purely quadratic one (azimuth scaling), whose Doppler rate $K_{\text {scl }}$ is the same for all ranges. After an inverse FFT in the azimuthal direction the signal is deramped (SPECAN), so that
TABLE I

Main System ANd PROCESSING PARAMETERS

\begin{tabular}{lc}
\hline Carrier frequency & $9.65 \mathrm{GHz}$ \\
Azimuth beamwidth $(3 \mathrm{~dB})$ & $0.33^{\circ}$ \\
System PRF & $3475 \mathrm{~Hz}$ \\
Sampling frequency & $150 \mathrm{MHz}$ \\
Height & $514 \mathrm{~km}$ \\
Ground velocity & $6800 \mathrm{~m} / \mathrm{s}$ \\
Mean look angle & $30.42^{\circ}$ \\
Maximum steering angle & $\pm 0.43^{\circ}$ \\
Burst duration & $0.2667 \mathrm{~s}$ \\
Imposed azimuth resolution & $16 \mathrm{~m}$ \\
\hline
\end{tabular}

after a final FFT the focusing is performed. The target is located in a frequency proportional to the scaling Doppler rate and its azimuth position $t_{0}$, i.e. $f_{\text {target }}=K_{\text {scl }} \cdot t_{0}$. Furthermore, with this approach also the azimuth image spacing can be selected to ease the combination of different subswaths.

The problem lies in the fact that if $\left|K_{\text {scl }}\right| \cdot \Delta t_{\text {scene }}>$ PRF aliasing will appear, where $\Delta t_{\text {scene }}$ is the length of the output scene. Commonly, this does not occur in ScanSAR, spotlight or sliding spotlight imaging modes, but becomes and important issue in the TOPS mode. There exist several possibilities to circumvent this problem:

- Apply the FFT in smaller blocks and discard after the FFT the non full resolution targets. A recombination will be necessary afterwards to combine the blocks. This turns out to be an inefficient solution.

- Another possibility is to increase the PRF by means of zero padding. This step should be carried out before the azimuth scaling operation, hence increasing the computation burden of the forthcoming steps. This option is not efficient either.

- A further option is to select a proper scaling range, so that $K_{\text {scl }}$ is small enough to avoid aliasing, which yields the following equation

$$
r_{\mathrm{scl}}>\frac{2 \cdot v^{2}}{\lambda \cdot \mathrm{PRF}} \Delta t_{\text {scene }}
$$

The problem in this case is that the scaling range can be far away from the swath, hence invalidating the possibility to perform azimuth scaling properly. For example, with the system parameters of TerraSAR-X shown in Table I and a target at $r_{0}=596.09 \mathrm{~km}$, the scene duration is $\Delta t_{\text {scene }}=1.58 \mathrm{~s}$, so that the obtained $r_{\text {scl }}$ using (1) is $1358.5 \mathrm{~km}$, i.e. more than 2 times $r_{0}$. The necessary zeropadding to accommodate for the stretching of the signal after azimuth scaling makes this approach also inefficient. Next Section provides an efficient solution to perform TOPS focusing, based on a new azimuth scaling approach.

\section{Proposed Processing based on ECS}

The block diagram of the proposed processor appears in Fig. 3. In order to accommodate the larger scene bandwidth, data are divided in azimuth blocks (sub-apertures), similarly as done for spotlight processing in [5]. After the division into subapertures, the processing for each sub-aperture is continued 


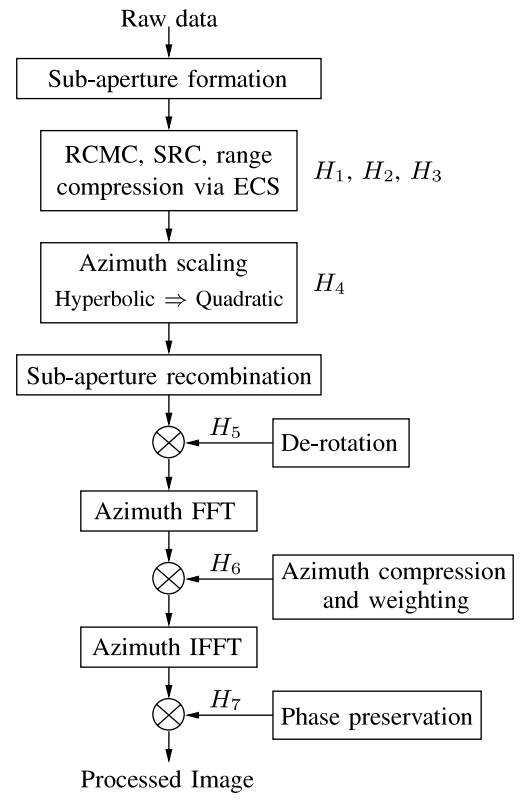

Fig. 3. Block diagram of the proposed processor for the TOPS imaging mode.

with the corresponding Doppler centroid. To prevent a poor processing result due to the block processing, the sub-apertures are formed with some overlap, e.g. 5\%. The steps of RCMC, secondary range compression (SRC) and range compression are carried out using the standard phase functions of ECS [2] (functions $H_{1}, H_{2}$ and $H_{3}$ ) for each sub-aperture. Once in the range-Doppler domain, a novel azimuth scaling is performed, named baseband azimuth scaling. This results in the removal of the hyperbolic azimuth phase and the replacement of the same with a quadratic phase shape using

$$
\begin{aligned}
H_{4}\left(f_{a}, r\right) & =\exp \left[\mathrm{j} \frac{4 \pi}{\lambda} r \cdot\left(\beta\left(f_{a}\right)-1\right)\right] \\
& \cdot \exp \left[-\mathrm{j} \frac{\pi}{K_{\mathrm{scl}}(r)} f_{a}^{2}\right],
\end{aligned}
$$

with

$$
\beta\left(f_{a}\right)=\sqrt{1-\left(\frac{\lambda f_{a}}{2 v}\right)^{2}} .
$$

The purely quadratic phase history is described by the scaling Doppler rate $K_{\text {scl }}(r)$. The scaling range is not constant (as in [2]), and it is not equal to the original range vector $r$ (as in [6]); it depends on range as described in equations (4) to (6):

$$
\begin{aligned}
K_{\mathrm{scl}} & =-\frac{2 v^{2}}{\lambda r_{\mathrm{scl}}(r)} \\
r_{\mathrm{scl}}(r) & =\frac{r_{\mathrm{scl} 0}}{r_{\mathrm{rot} 0}} r_{\mathrm{rot}}(r) \\
r_{\mathrm{rot}}(r) & =\frac{r_{\mathrm{rot} 0}-r}{1-r_{\mathrm{scl} 0} / r_{\mathrm{rot} 0}},
\end{aligned}
$$

where $r_{\text {sclo }}$ is a scaling range selected considering the desired azimuth image spacing, and $r_{\text {rot0 }}$ is the vector distance to the rotation center, as depicted in Fig. 4. The reason to use this range-dependent scaling range is explained later.

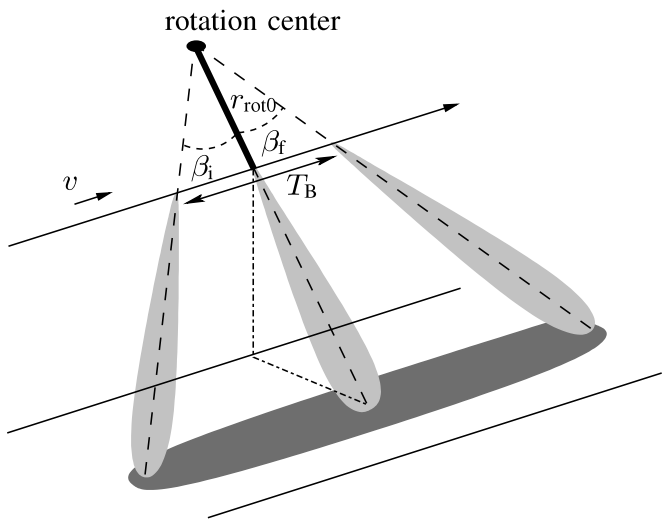

Fig. 4. Sketch showing the location of the rotation center and the rotation range in the TOPS imaging mode.

Since the azimuth scaling results in a displacement of the azimuth signals that are not located in the illumination center, a slight extension of the azimuth dimension is required. However, this extension usually falls within the range attained by the extension of the sub-aperture size to the next power of two. In the next step of the process, an azimuth IFFT is used for a transformation back to the azimuth/range-time, where the individual sub-apertures are assembled. However, the bandwidth of the signal still spans over the PRF. Therefore a demodulation can be carried out similarly as in [3] by using the following de-rotation function

$$
H_{5}(t, r)=\exp \left[-\mathrm{j} \pi K_{\mathrm{rot}}(r) \cdot\left(t-t_{\mathrm{mid}}\right)^{2}\right],
$$

where $t_{\text {mid }}$ is the scene center time. The effect on the signal due to this de-rotation function is depicted in Fig. 5. The chirp rate used in the de-rotation function depends on range and is given by

$$
K_{\text {rot }}(r)=-\frac{2 v^{2}}{\lambda r_{\text {rot }}(r)} .
$$

At this point, the effective chirp rate of the signal has been changed to $K_{\text {eff }}(r)=K_{\text {scl }}(r)-K_{\text {rot }}(r)$. Due to the fact that now the data spectrum for all targets is basebanded, matched filtering can be applied using

$$
H_{6}\left(f_{a}, r\right)=W\left(f_{a}\right) \cdot \exp \left[\mathrm{j} \frac{\pi}{K_{\text {eff }}(r)} f_{a}^{2}\right] .
$$

At this point, also azimuth sidelobe suppression can be easily performed by means of a weighting function $W\left(f_{a}\right)$. An

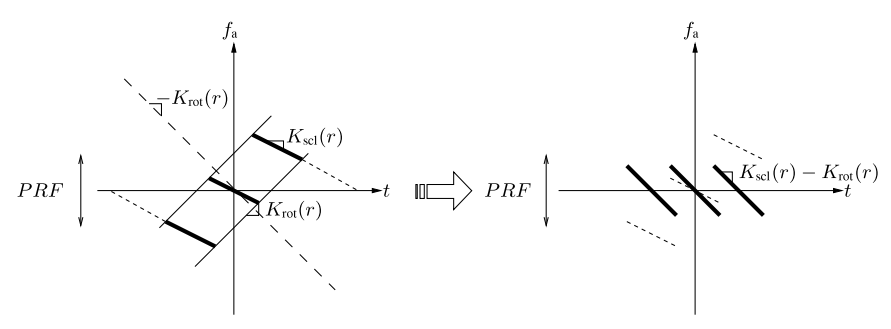

Fig. 5. Sketch showing how basebanding is performed by multiplying with a chirp function (long dashed line in left plot) in time-domain. 
inverse FFT results in a focused signal. However, for phase preserving processing data must be multiplied by the following phase function

$$
H_{7}(t, r)=\exp \left[\mathrm{j} \pi K_{\mathrm{t}}(r) \cdot\left(1-\frac{r_{\mathrm{scl} 0}}{r_{\mathrm{rot} 0}}\right)^{2} \cdot\left(t-t_{\mathrm{mid}}\right)^{2}\right],
$$

where

$$
K_{\mathrm{t}}(r)=-\frac{2 v^{2}}{\lambda \cdot\left(r_{\text {rot }}(r)-r_{\text {scl }}(r)\right)} .
$$

The need for a range-dependent scaling rate is explained in the following. The azimuth image sampling after using the azimuth scaling approach is given by

$$
\Delta x_{\text {final }}=\Delta x_{\text {orig }} \cdot\left(1-\frac{r_{\text {scl }}}{r_{\text {rot }}}\right) \text {. }
$$

where $\Delta x_{\text {orig }}$ is the original sampling equal to $v / \mathrm{PRF}$. One could think of using a constant scaling range $r_{\text {scl }}^{\prime}$, but then the rotation range changes as expressed by

$$
r_{\text {rot }}^{\prime}=r_{\text {rot }}-\left(r-r_{\mathrm{scl}}^{\prime}\right) \text {. }
$$

If (13) is used in order to properly baseband the signal when a constant scaling range is used, then the final azimuth image sampling will depend on range, as noted in (12). Therefore, since it is desired to have the same azimuth image sampling for all ranges and at the same time properly baseband all targets, the solution is to use a scaling and a de-rotation vector given by equations (5) and (6). Doing so, the azimuth image sampling is given by $\Delta x_{\text {final }}=\Delta x_{\text {orig }} \cdot\left(1-r_{\text {scl0 }} / r_{\text {rot0 } 0}\right)$, where $r_{\text {scl0 }}$ is selected according to the desired final azimuth image sampling and should be within the range of the respective subswath in order to minimize the needed extension of the sub-apertures. Note that in the TOPS acquisition mode $r_{\text {rot0 }}$ is negative, so that the new image sampling is larger than the original raw data sampling, which is desirable as the resolution is worse than in the stripmap case.

\section{RESULTS}

In order to validate the experimental processor, TOPS raw data of point targets were simulated. The main system and processing parameters appear in Table I. Fig. 6 shows the zeropadded contour-plot of the processed targets. The nine point targets have different range and azimuth positions corresponding to the same TOPS burst. They are arranged such that the targets in the corner positions limit a scene with the dimensions of $7.2 \mathrm{~km} \times 24.6 \mathrm{~km}$ in azimuth and range, respectively. The selected reference scaling range is $r_{\mathrm{scl} 0}=596091.37 \mathrm{~m}$, while the rotation range is equal to $r_{\text {rot } 0}=-120803.01 \mathrm{~m}$. Therefore, the final azimuth image spacing is $\Delta x_{\text {final }}=$ $11.61 \mathrm{~m}$. The maximum deviations of the measured resolutions from the theoretical values are $2 \%$ in azimuth and $1 \%$ in range. The phase preservation of the algorithm was also validated with point targets, and was found to be within $1^{\circ}$ accuracy. It is worth mentioning that the large Doppler centroid under which the targets at the edges of the burst are observed can impose strong requirements in the coregistration accuracy (due to the azimuth phase ramp [7]), above all at higher frequency bands, like X-band.
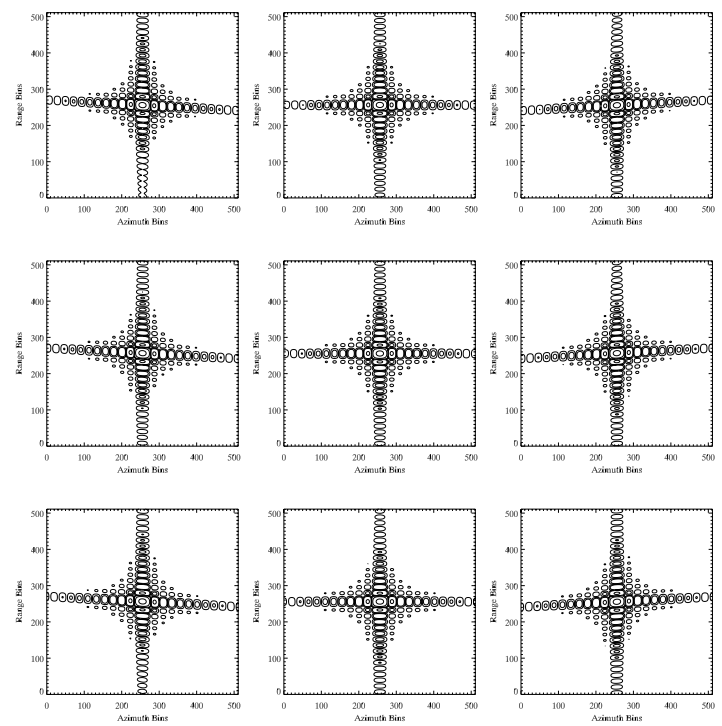

Fig. 6. Contour plots showing the processing result of nine point targets located at (top) near, (middle) mid, and (bottom) far range.

\section{CONCLUSION}

A phase preserving TOPS processor has been presented. It divides the raw data in azimuth sub-apertures to accommodate the scene bandwidth, while for the azimuth processing a new azimuth scaling approach allows an efficient focusing of the data. The baseband azimuth scaling also allows selecting the final azimuth image spacing, which is useful since it avoids the need for interpolations to recombine different subswaths. When implemented together with ECS, the overall focusing is carried out without interpolations, but just using FFTs and complex multiplications. For a detailed performance analysis of the TOPS imaging mode with TerraSAR-X refer to [8].

\section{ACKNOWLEDGMENTS}

The work in this project has been partially founded by ESA contract C20679/07/NL/CB.

\section{REFERENCES}

[1] A. Monti Guarnieri and C. Prati, "ScanSAR focusing and interferometry," IEEE Trans. Geosci. Remote Sensing, vol. 34, no. 4, pp. 1029-1038, July 1996.

[2] A. Moreira, J. Mittermayer, and R. Scheiber, "Extended chirp scaling algorithm for air- and spaceborne SAR data processing in stripmap and scanSAR imaging modes," IEEE Trans. Geosci. Remote Sensing, vol. 34, no. 5, pp. 1123-1136, Sept. 1996.

[3] F. De Zan and A. Monti Guarnieri, "TOPSAR: Terrain observation by progressive scans," IEEE Trans. Geosci. Remote Sensing, vol. 44, no. 9, pp. 2352-2360, Sept. 2006.

[4] A. M. C. Prati and F. Rocca, "SPOT mode SAR focusing with the $\omega-k$ technique," in Proc. IEEE International Geoscience and Remote Sensing Symposium (IGARSS'91), Espoo, Finland, July 3-6 1991, pp. 631-634.

[5] J. Mittermayer, A. Moreira, and O. Loffeld, "Spotlight SAR data processing using the frequency scaling algorithm," IEEE Trans. Geosci. Remote Sensing, vol. 37, no. 5, pp. 2198-2214, Sept. 1999.

[6] A. Moreira, R. Scheiber, and J. Mittermayer, "Azimuth and range scaling for SAR and ScanSAR processing," in Proc. IEEE International Geoscience and Remote Sensing Symposium (IGARSS'96), Lincoln, Nebraska, science and Remote Sensing
USA, May 27-31, 1996.

[7] R. Scheiber and A. Moreira, "Coregistration of interferometric SAR images using spectral diversity," IEEE Trans. Geosci. Remote Sensing, vol. 38, no. 5, pp. 2179-2191, July 2000.

[8] A. Meta, J. Mittermayer, U. Steinbrecher, and P. Prats, "Investigations on the TOPSAR acquisition mode with TerraSAR-X," in Proc. IEEE IGARSS 2007, Barcelona, Spain, July 23-27, 2007. 